ANARChISMUS UND LITERATUR 


\section{Anarchismus und Literatur}

Ein vergessenes Kapitel deutscher Literaturgeschichte zwischen 1890 und 1910 
CIP-Kurztitelaufnahme der Deutschen Bibliothek

Fähnders, Walter:

Anarchismus und Literatur : e. vergessenes Kap.

dt. Literaturgeschichte zwischen 1890 u. 1910 /

Walter Fähnders. -

Stuttgart : Metzler, 1987.

ISBN 978-3-476-00622-6

ISBN 978-3-476-03247-8 (eBook)

DOI 10.1007/978-3-476-03247-8

Dieses Werk einschließlich aller seiner Teile ist urheberrechtlich geschützt. Jede Verwertung außerhalb der engen Grenzen des Urheberrechtsgesetzes ist ohne Zustimmung des Verlages unzulässig und strafbar. Das gilt insbesondere für Vervielfältigungen, Übersetzungen, Mikroverfilmungen und die Einspeicherung und Verarbeitung in elektronischen Systemen.

(C) 1987 Springer-Verlag GmbH Deutschland

Ursprünglich erschienen bei J. B. Metzlersche Verlagsbuchhundlung und

Carl Ernst Poeschel Verlag GmbH in Stuttgart 1987 


\section{INHALTSVERZEICHNIS}

Vorbemerkung ....................... VII

Kapitel I:

Anarchismus, Naturalismus und Sozialdemokratie . . . . . . . . . . . . . .

Die Opposition der Jungen (S. 4) - Zur )Überwindung des Naturalismus (S. 8) - John Henry Mackay und die 'Anarchisten`(S. 13) - Gustav Landauer, der ’Todesprediger` und der 'Sozialist (S. 22)

Kapitel II:

Literatur der Individuellen Expropriation . . . . . . . . . . . . . . . . . .

Die Einbrecher-Presse von Conrad Fröhlich (S. 37) - Prämissen (S. 39) - Offensive: Individuelle Expropriation (S. 43) - Defensive: Die Opfer (S. 46) - Tabuverletzungen (S. 50)

\section{Kapitel III:}

Anarchistische Lyrik . . . . . . . . . . . . . . . . . . . . . . . .

'Explosionslyrik` von Conrad Fröhlich (S. 55) - Anarchistische und sozialistische'Marseillaisen`(S. 64) - Der ’erste Sänger der Anarchie؛ John Henry Mackay (S. 86) - Anarchistische 'Tendenzlyrik: Erich Mühsam (S. 97) - Proletarische, sozialistische und anarchistische Lyrik (S. 109) - Ein 'Internationales Rebellen-Liederbuch ‘(S. 117)

\section{Kapitel IV:}

Anarchismus und Décadence . . . . . . . . . . . . . . . . . . . . . . . .

Das 'dekadente Syndrom (S. 124) - Décadence und Proletariat (S. 126) - Dekadente 'Asozialität‘ (S. 129) - Bürgerliche Décadence-Kritik (S. 132) - Sozialistische DécadenceKritik (S. 134) - Anarchistische Décadence-Rezeption (S. 136) - Dekadente AnarchismusRezeption (S. 142) - Kurt Martens: 'Roman aus der Décadencer (S. 144) - Anarchismus und Satanismus bei Stanislaw Przybyszewski (S. 152) - 'Satans Kinder (S. 160)-Künstler und Terror (S. 166) - Décadence/Bürgertum/Anarchismus (S. 168)

Kapitel V:

Anarchismus und Boheme . . . . . . . . . . . . . . .

Gustav Landauer, Erich Mühsam und die 'Neue Gemeinschaft‘ (S. 172) - Boheme und Lumpenproletariat (S. 177) - Künstler und Kunst (S. 183) 
Ausblick:

Anarchismus und Surrealismus bei Benjamin und Caudwell . . . . . . . . . . 188

Anmerkungen ....................... 199

zur Vorbemerkung . . . . . . . . . . . . . . . . . . . . . . . 199

zu Kapitel I . . . . . . . . . . . . . . . . . . . . . . . . . . . . . . . . . . . . . . . 199

zu Kapitel II . . . . . . . . . . . . . . . . . . . . . . . . . . . 208

zu Kapitel III . . . . . . . . . . . . . . . . . . . . . . . . . . . . . 211

zu Kapitel IV . . . . . . . . . . . . . . . . . . . . . 226

zu KapitelV . . . . . . . . . . . . . . . . . . . . . 234

zum Ausblick . . . . . . . . . . . . . . . . . . . . . . . . . . . 238

Literaturverzeichnis . . . . . . . . . . . . . . . . . 240

Namenregister ................... 257 
Wie alle sozialen Bewegungen hat auch der Anarchismus seine Literatur, und der Anarchismus selbst ist zum Thema von Literatur geworden. Die Leitfrage dieser Arbeit lautet: was ist das für eine Literatur, die Anarchisten geschrieben haben, und wie erscheinen Anarchismus und Anarchisten in der Literatur (bestimmter) nicht-anarchistischer Autoren.

Dabei kann es hier nicht um eine Darstellung von Geschichte und Theorie des Anarchismus oder gar um eine Anarchismus-Theorie gehen. Der Anarchismus hat ja selbst etwas von Theorielosigkeit an sich, weil für ihn Theorie im Sinne eines geschlossenen Denkgebäudes durchaus etwas 'Archisches ist. Das heißt allerdings nicht, daß es keine durchgearbeiteten anarchistischen Theorien und keine reflektierte anarchistische Praxis gäbe: von Proudhon bis Stirner, von Bakunin bis Kropotkin, von Landauer bis Mühsam sind Modelle einer herrschaftsfreien Gesellschaftsordnung durchdacht und zum Teil erprobt worden; wo es geboten erscheint, werden sie in dieser Arbeit skizziert.

Der Forschungsstand zum Thema ist defizitär. Zwar existiert über den Anarchismus eine kaum mehr überschaubare Literatur, aber sie ist oft genug denunziatorisch und folgt dem Klischee vom Bombenwerfer, oder sie kommt apologetisch daher wie nicht selten in anarchistischen Darstellungen oder teilweise pseudokritisch und liquidatorisch wie in manchen marxistisch-leninistischen Arbeiten. In einem Forschungsbericht schreibt Peter Lösche 1977, daß »nur mit Vorbehalt von 'Erträgen der Forschung gesprochen werden (kann), wenn nach Interpretationen von Schriften und nach Biographien einzelner deutscher Anarchisten gefragt wird « [1]. Das ist auf die Geschichts- und Sozialwissenschaften gemünzt, gilt aber in noch größerem Ausmaß für die germanistische und literaturwissenschaftliche Forschung. Wenn Helmut Kreuzer 1968 feststellen mußte, daß die "Bedeutung « des Anarchismus » für die deutsche Literatur « bisher "kaum erkannt" sei [2], so gilt das in gewisser Weise bis heute. Zwar liegen mittlerweile einige Arbeiten über Gustav Landauer, Erich Mühsam und John Henry Mackay vor, aber das historische, literarische und personelle Geflecht von 'Anarchismus und Literatur bleibt zu entwirren.

Hier setzt die vorliegende Arbeit an. Sie konzentriert sich zwangsläufig auf einen allerdings markanten - Zeitraum im deutschen Kaiserreich, auf die Jahrzehnte vor und nach der Jahrhundertwende. Sie beginnt dort, wo in Deutschland die anarchistische Bewegung einen gewissen Aufschwung nimmt und wo auch in der bürgerlich-oppositionellen Literaturbewegung Veränderungen sich abzeichnen; und sie endet mit dem letzten Versuch vor dem 1. Weltkrieg, einen Neubeginn anarchistischer Gruppenbil- 
dung mit dem 'Sozialistischen Bund zu unternehmen - literarhistorisch umfaßt die Arbeit die Spanne zwischen der 'Úberwindung des Naturalismus` und dem `Aufbruch der Modernerseit dem Frühexpressionismus.

Die Literatur gerade dieses Zeitraumes zwischen 1890 und 1910 ist, wie die Arbeitsergebnisse wohl zeigen, von anarchistischen Positionen und von Auseinandersetzungen mit dem Anarchismus in nicht geringem Maße mitgeprägt. Das gilt für die Krisenphase des Naturalismus, als dieser sich von der sozialistischen Bewegung entfernt und individualistische Stellungen unterschiedlichster Provenienz, den Anarchismus in der Stirner-Nachfolge eingeschlossen, bezieht (Kap. I). Das gilt für das weite Feld anarchistischer Agitation, die hier an einem Extrembeispiel, der Literatur der individuellen Expropriation`, analysiert wird (Kap. II), sowie für die umfängliche anarchistische Lyrik von Autoren proletarischer und bürgerlicher Herkunft, die ideologisch wie ästhetisch auf ihre Innovationen, auf Traditionszusammenhänge und Rezeptionsbedingungen hin untersucht werden (Kap. III). Auf den ersten Blick überraschende Nahtstellen bestehen zwischen Anarchismus und Décadence: so gesellschaftsfern waren, wie im IV. Kapitel aufgezeigt ist, die künstlichen Paradiese der Décadence nicht, als daß sie sich in ihrem Destruktionsarsenal den Anarchismus hätte entgehen lassen. Parallel dazu werden die engen Verflechtungen zwischen Anarchismus und Boheme in klassenanalytischer, 'lebenspraktischer' und personeller Hinsicht skizziert (Kap. V). Der 'Ausblick reflektiert die gänzlich veränderten Literaturbedingungen der 20er und 30er Jahre, unter denen Affinitäten zwischen Anarchismus und ästhetischer Avantgarde thematisiert werden, und zwar anhand der Surrealismus-Interpretationen von Walter Benjamin und Christopher Caudwell.

Der defizitäre Forschungsstand hat zwangsläufig zu thematischen Einschränkungen führen müssen; so wäre es mit Sicherheit von Gewinn, weitere Profile exponierter anarchistischer Zeitschriften gerade unter literaturwissenschaftlichen Aspekten zu entwerfen, den Blick auf den deutschsprachigen Exil-Anarchismus in den USA vor dem 1. Weltkrieg zu richten [3] und die umfangreiche anarchistische Memoirenliteratur aufzuarbeiten. Insofern ist die vorliegende Arbeit ein erster größerer Versuch, den Zusammenhängen zwischen Anarchismus und Literatur in dem genannten Zeitraum nachzuspüren. Daß dabei oft ausführlich zitiert wird und die Deskription einen gewissen Raum einnimmt, liegt daran, daß viele Quellen nur schwer zugänglich sind und mit der Untersuchung auch Einblick ins untersuchte Material selbst gegeben werden soll. Ob beides lesbar ist, mögen Leserin und Leser beurteilen. 
Die vorliegende Arbeit ist 1985 vom Fachbereich Sprache, Literatur, Medien der Universität Osnabrück als Habilitationsschrift angenommen worden; sie erscheint hier in überarbeiteter Fassung. Den Druck hat dankenswerter Weise die DFG durch einen Zuschuß gefördert.

Ich danke allen, die mich bei meiner Arbeit unterstützt haben - namentlich Heiner Becker (Münster/Amsterdam), Klaus Garber (Osnabrück), Helmut Glück (Osnabrück/ Kairo), Helga Karrenbrock (Osnabrück), Lothar Knapp (Osnabrück), Christiane Krause (Osnabrück), Hartmut Schilling (Osnabrück).

Alle Zitate, einschließlich der Hervorhebungen, folgen der Originalschreibweise; lediglich Umlaute und 'ss` oder 'sz'(für 'ßs) sind vereinheitlicht worden.

Osnabrück, April 1987

Walter Fähnders 